\title{
Working in Hydrodynamics with Yogiro Hama
}

\author{
F. Grassi \\ Instituto de Física, Universidade de São Paulo, C. P. 66318, 05315-970 São Paulo, SP, Brazil
}

Received on 29 September, 2006

\begin{abstract}
I present a brief overview on the motivation for performing relativistic nuclear collisions and the justification for using hydrodynamics to describe them. The contribution of Prof. Hama to the field of hydrodynamics and his efforts to form a group working on this subject are outlined.
\end{abstract}

Keywords: Hydrodynamics; Nuclear collisions; Quark-gluon plasma

\section{MOTIVATION}

Hydrodynamics has many applications: formation and propagation of waves, wheather forecast, lift on airplane wing, black hole or dense stellar object collapse, primordial universe expansion, etc. How and why is hydrodynamics used in our field, the physics of relativistic hadronic or nuclear collisions?

It was predicted in the mid-seventies, that a new state of matter might exist, the quark-gluon plasma. This can be understood in the following way. When adding heat to ice, it undergoes a phase transition to water and adding more heat, this water undergoes a phase transition to vapor. In the same way, heating and compressing nuclei, they would change from state and melt into their subconstituents, the quarks and gluons. This new state of matter is called quark-gluon plasma. The prediction is based upon a property of the interactions between quarks and gluons, called assymptotic freedom (for its discovery, Gross, Politzer and Wilczek shared the Nobel Prize in 2004). Due to this property, it was conjectured [1] that at high densities or temperatures, quarks become free. The first phase diagram representing the phase transition from quarks confined inside hadrons to a state of free quarks appeared I believe in [2].

This prediction lead to many theoretical and experimental efforts in the study of relativistic nuclear collisions. On the experimental side, various accelerators have been used, reaching energies each time higher. A summary is shown in Table 1.

\begin{tabular}{|c|c|c|c|}
\hline Beginning & Accelerator & $\begin{array}{c}\text { Beam } \\
\text { energy } \\
\text { GeV.A }\end{array}$ & $\begin{array}{c}\text { Center of mass } \\
\text { energy } \\
\text { GeV.A }\end{array}$ \\
\hline 1986 & $A G S$ & $\sim 15$ & $\sim 5$ \\
1986 & SPS & $\sim 200$ & $\sim 20$ \\
2000 & RHIC & - & $\sim 200$ \\
2009 & LHC & - & $\sim 5500$ \\
\hline
\end{tabular}

Table 1: summary of accelerators used for relativistic nuclear collisions.

On the theoretical side, the phase diagram became more complicated. First, depending on the u,d and s quark masses, the deconfinement could be a first order phase transition, a second order phase transition or a mere "crossover" (see for example [3]). Second, beside the quark gluon plasma, many new possible phases of matter have been suggested: superconducting quark matter of various types, Bose-Einstein condensates of pions or kaons, (more or less stable) strange quark matter droplets called strangelets, atoms called Metastable Exotic Multistrange ObjectS or MEMOS for short, that would have some $\Lambda$ or $\Xi$ particles as well as nucleons, etc. The situation now looks much more complicated than for the ice-water case? In reality not so much: water in its solid (ice) phase can exist in many different phases, with new ones being discovered all the time (see e.g. [4] and more modern versions of the water phase diagram).

It is natural to ask why it is important to discover and study the quark-gluon plasma. This form of matter must have been present in the early universe, when it was much hotter and denser than today. It may exist as well in the core of neutron stars. There may exist stars made of quark matter with properties similar to that of neutron stars, or even strange stars made of strange quark matter and with properties different from that of neutron stars. The quark-gluon plasma may exist also in cosmic rays. Colliding nuclei at high energy with accelerators is a systematic way of (hopefully) creating quark-gluon plasma and learn about its properties. This in turn will permit us to understand better the universe [5].

As one perfoms relativistic nuclear collisions, the number of created particles increases with increasing accelerator energy. Order of magnitudes are shown in Table 2.

\begin{tabular}{|c|c|c|c|}
\hline AGS & \multicolumn{2}{|c|}{ SPS } & RHIC \\
$\mathrm{Au}+\mathrm{Au}$ & $\mathrm{S}+\mathrm{S}$ & $\mathrm{Pb}+\mathrm{Pb}$ & $\mathrm{Au}+\mathrm{Au}$ \\
\hline$h^{-} \sim 176$ & $h^{-}=103 \pm 5$ & $h^{-} \sim 680$ & $N_{c h}=4100 \pm 100 \pm 400$ \\
EMU01 & NA35 & NA49 & Phobos@130 GeV \\
\hline
\end{tabular}

Table 2: number of negative or charged particles created at various energies.

The question then arises as to how to describe the creation, evolution and final stage of the matter created in these nuclear collisions. In the so-called microscopic models, each colliding parton or nucleon is followed. However, beside the numerical complexity and theoretical uncertainties, the transition from quark matter to hadronic matter is not incorporated. On the other extreme, Landau suggested very early, in 1953, that high energy collisions might be described using hydrodynamics [6]. Today, in the relativistic nuclear collisions performed, the role of hydrodynamics has become more apparent as we are going to discuss. 


\section{RETROSPECTIVE OF THE USE OF HYDRODYNAMICS IN RELATIVISTIC NUCLEAR COLLISIONS}

Initially the transverse momentum (i.e. perpendicular to the beam axis) of particles is small (in average) and due to collisions and expansion, increases. Therefore the distribution of transverse momentum of particles provides information about the fluid temperature and velocity when these particles are emitted. In general it is supposed that this emission is sudden and it is called freeze-out.

We can obtain an overview on the use of hydrodynamics in relativistic nuclear collisions by looking at transverse momentum distributions in the Proceedings of the Quark Matter Conferences (QM for short), the main ones in the field, which until recently occurred every 1.5 year and now are annual. Immediately after the first really ultrarelativistic nuclear collisions were performed, at QM87, NA35 showed that their negative particle spectrum could be adjusted to a thermal model [7]. Though it worked, noone could understand the meaning of having two temperatures in the fit. At QM88, the same collaboration [8] used a hydrodynamics inspired parametrization [9] and showed that it reproduced the transverse spectra of negative particles for $\mathrm{O}+\mathrm{Au}$ and $\mathrm{S}+\mathrm{S}$ collisions and heavier particles for O+Au. At QM90, U.Heinz [10] using a model of the type [9] studied spectra from various experiments. At QM91, NA35 used a microscopic code called Venus to fit their kaon transverse with some success [11]. At QM93, it was emphasized by D.Strottman that in various cases hydrodynamics and microscopic models lead to similar predictions [12]. At QM95, it was shown that with the heavier nucleus collisions, $\mathrm{Pb}+\mathrm{Pb}$, transverse momentum spectra of various particle types could also be reproduced with a hydrodynamical inspired parametrization [13]. Until QM95, a single freeze out temperature was used. After QM97, it became standard to assume that two freeze outs occur: first chemical freeze out or freezing of inelastic interactions (particle numbers are fixed) then thermal freeze out or freezing of elastic interactions (spectrum shapes are fixed) [14]. At QM97, G.Odyniec pointed out the difficulties of microscopical models to reproduce all data in particular on strangeness enhancement [15]. This was reinforced at QM99 [16]. While on the theoretical side, it was emphasized that hadronic microscopic models should not be used alone for collisions involving heavy nuclei at SPS energies or higher, due to the high densities involved [17]. RHIC result interpretation marks the beginning of the real general acceptance of hydrodynamics as a tool to describe relativistic nuclear collisions: by QM01, it was already realized that simplified hydrodynamics with boost invariant longitudinal expansion predicted the correct behavior of a variable called elliptic flow [18] (as well as reproduces data on transverse spectra of a variety of particles $[19,20]$ ) while other microscopic models fail to achieve this [19]. From then on, more sophisticated hydrodynamical codes have been developped in particular by Prof. Hama's group as will be explained in more detailed.

\section{CONTRIBUTION OF PROF. HAMA'S GROUP TO THE DESCRIPTION OF RELATIVISTIC NUCLEAR COLLISIONS USING HYDRODYNAMICS}

In this section, I concentrate on works on hydrodynamics done in Prof. Hama's group after the beginning of the runs at AGS and SPS, i.e. the beginning of the ultrarelativistic nuclear collisions. Works before this period are reviewed by F. Navarra in these proceedings.

Interferometry is a method that allows to determine the space and time dimensions of an emitting source. It is based on the probability to detect simustaneously two particles.

In the mid-fifties, interferometry was used to measure stellar radii. In 1960, Goldhaber and collaborators suggested its use in $p-\bar{p}$. In 1987, the first data on interferometry in $\mathrm{O}+\mathrm{Au}$ appeared. The same year, Sandra Padula defended her thesis on interferometry, under the supervision of Prof. Hama, emphasizing the role played by the source expansion [21]; today, this looks trivial but at the time it was not. Later on, Sandra worked as a posdoc in Berkeley, with M.Gyulassy, studying the role of resonance decays in interferometry measurements [22]. She then became a professor at the "Instituto de Física Téorica" of the UNESP university and continues working on the use of interferometry in relativistic hadronic and nuclear collisions.

In 1984, Fernando Navarra defended his M.Sc. thesis in hydrodynamics, under the supervision of Prof. Hama [23] and went on to do his Ph.D. in Marburg-Germany, with R.Weiner, which he completed in 1989 . He then became a professor at the Universidade de São Paulo and worked on many different subjects: the IGM model, minijets, $J / \Psi$ particles, pentaquarks, Color Glass Condensate, etc.

In 1992, Fernando and Prof. Hama predicted that the freeze out temperature should decrease with center-of-mass energy [24]. At QM01, Xu and Kaneta observed that this was indeed the case at RHIC energy [25].

In 1995, Prof. Hama with his long-time collaborator Takeshi Kodama and myself, developped the model of continuous emission of particles [26], as a more physical alternative to (sudden) freeze-out. This lead to various other works trying to improve particle emission, in collaboration with L.Csernai [27] and later with Y.Sinyukov [28]. More direct applications to analyze data were done with the doctorate student and then posdoc Otavio Socolowski Jr. In particular it was shown that the inclusion of continuous emission improves the comparison of interferometry data with hydrodynamical predictions [29].

Between 1995-97, Samya Paiva defended her Ph.D. thesis under the supervision of Prof. Hama and wrote various papers on fluctuations in p-p collisions [30]. In particular, they argued that the inclusion of fluctuations in the initial conditions was important to predict observables. So this was perhaps the motivation to initiate the development of the code SPheRIO (see later).

Celso Barros obtained his M.Sc. in 1995 and his Ph.D.Thesis in 1991, on hyperon polarization [31], under the supervision of Prof. Hama. His work was motivated by a proposal by Prof. Hama and T. Kodama [32] that part of the 
(anti)hyperon polarization comes from its interaction with the medium that surrounds it.

Nelmara Arbex made her M.Sc. with C.Nemes at USP in 1990, obtained her Ph.D. on hydrodynamics in Marburg with R. Weiner and worked as a posdoc with Prof. Hama. In 2001, Nelmara, Prof. Hama, Otavio and myself, made a work on the influence of separate chemical and thermal freeze outs on the fluid evolution [33]. Though we were the first to point out this, soon after there appeared more detailed works on this topic.

In 2003, T.Csörgo, made with Prof. Hama, T.Kodama [34] and myself [35], a search for exact solutions of the hydrodynamical equations in special cases.

In 2001, we started an effort that we still persue: the development of a code to solve the equations of relativistic hydrodynamics to describe relativistic nuclear collisions. It is based on a method called Smooth Particle Hydrodynamics and so the code became known as SPheRIO, from Smooth Particle hydrodynamical evolution of Relativistic Ions (thanks to T.Osada!) The method used allows to incoporate any kind of initial conditions, even highly irregular ones, which permits us to do event-by-event hydrodynamics. For the time being, we are the only group capable of this.

The first version of the code was mostly written by the posdoc Takeshi Osada [36]. Later on, many persons joined in. Otavio Socolowski Jr. included interferometry calculations, continuous emission, equation of state with crossover $[37,38]$. Rone Andrade, then a M.Sc. student and now a doctorate student, incorporated elliptic flow calculations [39] and is now improving the continuous emission part of the code. WeiLiang Quian, a posdoc, is including an equation of state with strangeness so we can compare with more data. Arthur L.V.R. dos Reis, doctorate student, will incorporate Color Glass Condensate initial conditions.

The SPheRIO code has now gained a good international acceptance, with T. Kodama presenting orally results at QM01 and Prof. Hama at QM05, as well as T. Hirano mentioning our code in his review talk at QM04. Lastly, at QM06, T. Kodama showed how to include dissipation in SPheRIO as well as preliminary results.

\section{CONCLUSION}

There is big effort undergoing in the world to discover a new phase of matter called Quark-Gluon Plasma, by performing very high energy collisions of heavy nuclei, in particular at RHIC. Hydrodynamics has been established as a good tool to study these collisions. Prof. Hama has oriented many students on hydrodynamics, worked on various improvements and applications. This has lead to the formation of a group in Brazil, ready to participate of the search for the Quark-Gluon Plasma.

The pdf file of the talk I gave for Prof. Hama's 70th birthday, including pictures and figures, can be found at http://fma.if.usp.br/ grassi/
[1] J. C. Collins and M. J. Perry, Phys. Rev. Lett. 34, 1353 (1975).

[2] N. Cabibo and G. Parisi, Phys. Lett. B 59, 67 (1975).

[3] F. Karsch Nucl. Phys. A 698, 199c (2002).

[4] H. B. Callen "Thermodynamics", Wiley International Edition, chapter 9.

[5] Committee on the Physics of the Universe, National Research Council, "Eleven Science Questions for the New Century": What Are the New States of Matter at Exceedingly High Density and Temperature?

[6] L. D. Landau Izv. Akad. Nauk, SSSR 17, 51 (1953); S. Z. Belenkij and L. D. Landau Usp. Fiz. Nauk 56, 309 (1955); in "Collected papers of L. D. Landau" (ed. D.Ter-Haar, Pergamon, Oxford, 1965) 569 and 665.

[7] A. Bamberger et al. (NA35) Z. Phys. C 38, 85 (1988) (figure 11).

[8] A. Bamberger et al. (NA35) Nucl. Phys. A 498, 133c (1989) (figures 5 and 6).

[9] P. J. Siemens and J. O. Rasmunssen, Phys. Rev. Lett. 42, 880 (1979); D. Hahn and N. K. Glendenning Phys. Rev. C 37, 1053 (1988); K. S. Lee and U. Heinz Z. Phys. C 43, 425 (1989).

[10] K. S. Lee, E. Schnedermann, J. Sollfrank, and U. Heinz, Nucl. Phys. A 525, 523c (1991) (figures 1 and 2).

[11] J. Baechler et al. (NA35), Nucl. Phys. A544, 609c (1992) (figures 4 to 7 ).

[12] D. Strottman, Nucl. Phys. A 566, 245c (1994) (figure 3).

[13] I.G. Bearden et al. (NA44), Nucl. Phys. A 610, 175c (1996).

[14] P. Braun-Munziger and J. Stachel, Nucl. Phys. A 638, 3c (1998); U.Heinz, ibid. 357c.

[15] G. Odyniec, Nucl. Phys. A 638, 135c (1998) (figure 8).
[16] F. Antinori et al (WA97), Nucl. Phys. A661, 130c (1999) (figure 9).

[17] B. Müller, Nucl. Phys. A 661, 272c (1999).

[18] R. Snellings et al. (STAR), Nucl. Phys. A 698, 193c (2002) (figure 5); P.Kolb and U. Heinz nucl-th/0305084 published in "Quark Gluon Plasma 3", World Scientific.

[19] U. Heinz and P. Kolb, Heavy Ion Phys. 15, 205 (2002) (figure $1)$.

[20] D. Teaney, J. Lauret and E.V. Shuryak nucl-th/0110037 (figure 19).

[21] Y. Hama and S. Padula, Phys. Rev. D 37, 3237 (1988).

[22] M. Gyulassy and S. Padula, Phys. Lett. B 217, 181 (1989).

[23] Y. Hama and F. Navarra, Phys. Lett. B 129, 251 (1983); Z. Phys. C 26, 465 (1984).

[24] Y. Hama and F. Navarra, Z. Phys. C 53, 501 (1992).

[25] N. Xu and M. Kaneta, Nucl. Phys. A 698, 306 (2002).

[26] F. Grassi, Y. Hama and T. Kodama, Phys. Lett. B 355, 9 (1995); Z. Phys. C 73, 153 (1996).

[27] Cs. Anderlik, L.P. Csernai, F. Grassi, W. Greiner, Y. Hama, T. Kodama, Zs.I. Lazar, V. Magas, and H. Stoecker, Phys. Rev. C 59, 3309 (1999); Phys. Lett. B 459, 33 (1999); Nucl. Phys. A 661, 596 (1999).

[28] Yu. M. Sinyukov, S. V. Akkelin, and Y. Hama, Phys. Rev. Lett. 89, 052301 (2002)

[29] F. Grassi, Y. Hama, S. Padula, and O. Socolowski Jr., Phys. Rev. C 62, 044904 (2002).

[30] S. Paiva, Y. Hama, and T. Kodama, Phys. Rev. C 55, 1455 (1997); Y. Hama, T. Kodama, and S. Paiva, Phys. Rev. Lett. 78, 3070 (1997). 
[31] C. Barros Jr. and Y. Hama, Phys. Rev. C 63, 1 (2001).

[32] Y. Hama and T. Kodama, Phys. Rev. D 48, 3116 (1993).

[33] N. Arbex, F. Grassi, Y. Hama, and O. Socolowski Jr., Phys. Rev. C 64, 64906 (2001).

[34] T. Csörgo, Y. Hama, and T. Kodama, Phys. Rev. C 67, 1 (2003).

[35] T. Csörgo, F. Grassi, Y. Hama, and T. Kodama Phys. Lett. B 565, 107 (2003).

[36] C. E. Aguiar, T. Kodama, T. Osada, and Y. Hama, J. Phys. G 27, 75 (2001); T. Kodama, C. E. Aguiar, T. Osada, and Y. Hama,
J. Phys. G 27, 557 (2001); C. E. Aguiar, Y. Hama, T. Kodama, and T. Osada Nucl. Phys. A 698, 639 (2002).

[37] O. Socolowski Jr, F. Grassi, Y. Hama, and T. Kodama, Phys. Rev. Lett. 93, 182301 (2004).

[38] Y. Hama, R. Andrade, F. Grassi, O. Socolowski Jr., T. Kodama, B. Tavares, and S. Padula, Nucl. Phys. A 774, 169 (2006).

[39] R. Andrade, F. Grassi, Y. Hama, T. Kodama, and O. Socolowski Jr. Phys. Rev. Lett. 97, 202302 (2006). 